\title{
Analysis for Crime Scene Investigation and Reconstruction
}

\author{
Samir Kumar Bandyopadhyay* \\ Department of Computer Science and Engineering, India \\ *Corresponding author: Samir Kumar Bandyopadhyay, Department of Computer Science and Engineering, India
}

Submission: 眥 March 26, 2018; Published: 䟧 April 24, 2018

\section{Introduction}

The Oxford Dictionary defines 'Crime' as 'an action or omission which constitutes an offence and is punishable by law'. The criminal proceedings that take place can broadly be classified into 3 basic phases-Investigation, Inquiry and Trial. Crime Scene Investigation and thereby Reconstruction in its turn particularly involves 3 basic phases. They are
a. Defining the crime scene
b. Processing the crime scene
c. Information collection from and about the crime scene

Blood was legally procured from Kolkata Municipal Pig Slaughter House, Tangra Kolkata, India. Given that fresh blood coagulates over time, 1100IU of Heparin Injection was added to fresh pig blood to preserve the colloidal consistency of blood. It might be interesting to mention that adding anticoagulant does not alter the viscosity and specificity of the Non-Newtonian fluid, blood. The research work is particularly aimed at improving the process of crime scene investigation and hence reconstruction. The University of Calcutta along with Kolkata Police and PG Hospital has been doing jointly the work for crime scene investigation and reconstruction.

A reference database of bloodstain drip pattern on different types of fabric (i.e. fabrics made from natural fiber, man-made fiber, fabrics used for industrial purposes), is created. A bloodstain pattern is defined as 'a grouping or distribution of bloodstains that indicate through regular or repetitive form, order, or arrangement the manner in which the pattern was deposited'. Based on the different case studies analysis we are of the view that of the different types of bloodstain patterns, the most common stain patterns visible at the crime scene, particularly in the case where the victim was found to suffer blunt force injuries, are saturation, impact, cast off and transfer stain patterns. A statistical analysis highlighting if there is a statistically significant difference in the stain patterns cast when the dosage of anticoagulant is varied shall be carried out Weapon transfer stains in a crime scene are often difficult to interpret. Given the large number of hammer hit events that take place each year, this work is largely aimed at analyzing the different factors that influence and distort regular hammer transfer stains. Different physical mechanisms under identical conditions influence the formation of distinct transfer stain patterns. This is the basic highlight of this study.

Many image processing techniques have been developed over the past two decades to help forensic scientist in detection of footprint image boundary. Most studies conducted have proven that measurement of parameters may help detection of crime scenario. Computer-aided recognition systems for footprint analysis have been the focus of several research endeavours and it based on the idea of processing and analysing Footprint images for a quick and accurate recognition. Footwear impressions are among the most commonly found evidence at crime scenes and present more frequently than finger prints. Identification is based on the physical match of random individual characteristics of the shoe has acquired during its life. The basic experiments on different materials pattern analysis Locard's exchange principle states that "every time an individual makes contact with another person, place or thing, it results in an exchange of physical materials" [1]. The study of evidence at a crime scene is particularly based on this principle. Bloodstain pattern analysis is defined as 'the scientific study of the static consequences resulting from dynamic blood shedding events' [2].

The detailed study of bloodstain patterns obtained from a crime scene could prove to be invaluable evidence for part/full crime scene reconstruction, in testing the credibility of the statements of the victim, suspect, bystander/eyewitness (if any). As per the International Association of Bloodstain Pattern Analysts (IABPA), a bloodstain pattern is defined as 'a grouping or distribution of bloodstains that indicate through regular or repetitive form, order, or arrangement the manner in which the pattern was deposited' [3]. Based on the different case studies presented at the IABPA conference [4] the authors are of the view that of the different types of bloodstain patterns, the most common stain patterns visible at 
the crime scene, particularly in the case where the victim was found to suffer blunt force injuries, are saturation, impact, cast off and transfer stain patterns.

In the IABPA Conference held in Tucson, Arizona, 2004, Peter Lamb presented the investigation report of the late night assault of a young man who was intoxicated at the time of attack and could only recollect part of the savagery that he had been subjected to [5]. Due to rain drop that had soaked the garment at the time of the assault it was difficult to examine the bloodstains on the soaked garment. However there was evidence of kicking and stomping. Based on the evidence the case finally proceeded for trial and the accused was proved guilty and hence imprisoned [5]. In his review of the Windsor city homicide case Scott Lamont pointed out that barefoot transfer impressions and footwear transfer impressions were found on the floor. Foot morphology confirmed that the prints were left by the suspect who was wearing boots [6].

Once the Crime Scene is broadly defined, the next phase of the Investigation process deals with 'Processing of the Crime Scene'. At the very onset, the Crime Scene Investigator establishes contact with the Law Enforcement Officer in charge of the crime scene. Also he/she makes a list of other people such as other law enforcement officers, coroner's personnel, public safety personnel, civilians and newspaper reporters etc. who have or have had access to the crime scene in question. The next step towards processing a crime scene is Securing the scene by use of police line tape or other means in order to prevent unwanted access to the scene by casual passer-by, people with malicious intentions etc. The scene is secured in order to leave all evidence at the crime scene undisturbed by wandering individuals. Once secured, the scene is subjected to initial overall survey by investigating officials. At this point, the officials leave all evidence undisturbed and develop initial theories based on apparent understanding of the crime scene. The officials also mark out potential evidence in the initial walk through phase. The first responders as also enforcement officials take into account the entry /exit points in the scene that require attention. This phase also requires the officials to make a list of equipment and precautions that the officials would need to take in order to document as also search the crime scene.

\section{References}

1. Welding S (2012) Locard's exchange principle. Forensic Handbook.

2. Nordby JJ (2006) Final Analysis Forensics.

3. Scientific Working Group on Bloodstain Pattern Analysis (2009) Scientific working group on bloodstain pattern analysis: recommended terminology. FBI-Standards and Guidelines.

4. http://www.iabpa.org/journal

5. (2004) The use of BPA modeling in investigations. Peter Lamb, p. 8.

6. (2004) City of windsor homicide case. Scott Lamont, p. 11.
Creative Commons Attribution 4.0 International License

For possible submissions Click Here
Submit Article

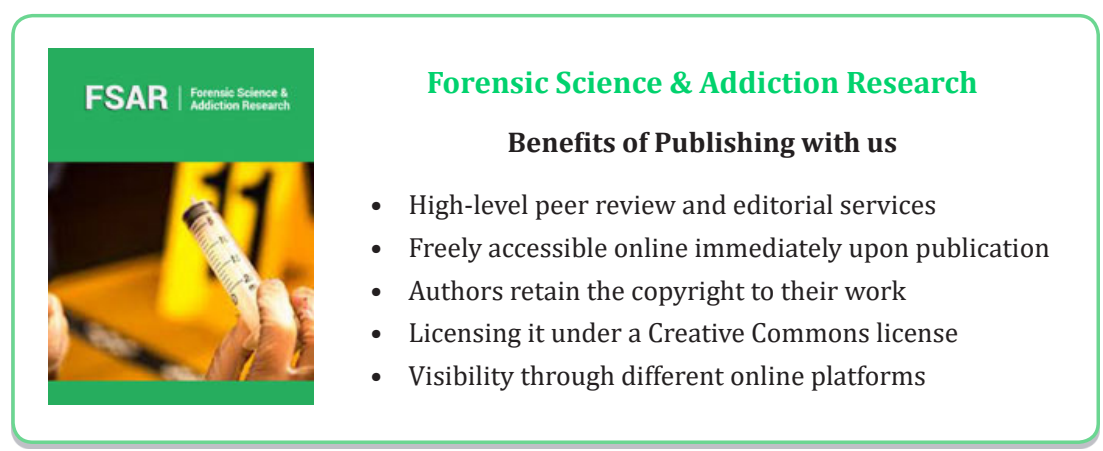

